\title{
Les Acadiens à Belle-île-en-Mer : une expérience originale d'intégration en milieu insulaire à la fin du XVIIIe siècle
}

\section{Christophe Cerino}

\section{(apenEdition Journals}

\section{Édition électronique}

URL : http://journals.openedition.org/abpo/1472

DOI : $10.4000 / a b p o .1472$

ISBN : 978-2-7535-1489-8

ISSN : 2108-6443

Éditeur

Presses universitaires de Rennes

Édition imprimée

Date de publication : 20 mars 2003

Pagination : 115-124

ISBN : 978-2-86847-811-5

ISSN : 0399-0826

\section{Référence électronique}

Christophe Cerino, "Les Acadiens à Belle-Île-en-Mer : une expérience originale d'intégration en milieu insulaire à la fin du XVIIIe siècle », Annales de Bretagne et des Pays de l'Ouest [En ligne], 110-1 | 2003, mis en ligne le 20 mars 2005, consulté le 23 avril 2019. URL : http://journals.openedition.org/ abpo/1472 ; DOI : 10.4000/abpo.1472 


\title{
Les Acadiens à Belle-Île-en-Mer : une expérience originale d'intégration en milieu insulaire à la fin du XVIII ${ }^{\mathrm{e}}$ siècle
}

\author{
Christophe CERINO \\ Docteur en histoire moderne \\ ATER, Université de Bretagne sud (Lorient)
}

En 1755, le déclenchement du " grand Dérangement " précipite les Français d'Acadie dans une longue diaspora. Pendant plusieurs décennies, des familles entières sont ballottées depuis les colonies de NouvelleAngleterre et (ou) les camps d'internement métropolitains britanniques jusqu'aux villes portuaires de France, qui deviennent elles-mêmes des points de départ pour de nouvelles tentatives d'établissement dans le Poitou, en Bretagne, en Guyane, à Saint-Domingue et aux Malouines ${ }^{1}$. Aux lendemains de la guerre de Sept Ans, près de 4200 personnes sont ainsi dispersées entre Boulogne, Le Havre, Cherbourg, Saint-Malo, Morlaix, Lorient, La Rochelle, Rochefort et Bordeaux, et pensionnées par le roi à raison de six sols par jour dans l'attente d'une installation convenable dans le royaume ${ }^{2}$.

Bien loin de l'Acadie, de l'autre côté de l'Atlantique, la guerre n'a pas épargné Belle-Ille-en-Mer. Ayant connu, après la bataille des Cardinaux en novembre 1759, le blocus de 1760, la présence en garnison des troupes royales (environ 2500 hommes), le siège de la citadelle et deux années d'occupation anglaise, l'île sort dévastée du conflit. En parallèle à un début d'exode, les principales ressources insulaires, à savoir la culture du froment et la pêche sardinière, sont totalement ébranlées, tandis que s'impose une profonde reconstruction de l'agrosystème médiéval, déjà défaillant depuis le début du XVIII ${ }^{\mathrm{e}}$ siècle $^{3}$.

1. LAUVRIERE, Émile, La tragédie d'un peuple. Histoire du peuple acadien de ses origines à nos jours, Paris, 1924, p. 181 à 270.

2. PITRE, Marie-Claire, Condow, James, The deportation of the Acadians, Environment Canada Parks, 1986, 16 p.

3. Pour une appréciation des conséquences économiques et sociales des conflits à Belle-Île au XVIII ${ }^{\mathrm{e}}$ siècle, nous nous permettons de renvoyer à notre thèse : Sociétés insulaires, guerres maritimes et garnisons. Belle-Île-en-Mer au siècle de Louis XV, Rennes 2, 2001, dir. Claude NiERES, $1475 \mathrm{p}$. 
Les États de Bretagne, qui ont la charge de Belle-Île en tant « qu'engagistes ${ }^{4}$ " du domaine de la Couronne, investissent plus de 200000 livres entre 1763 et 1766, pour rétablir l'activité économique dans les campagnes. Ils lancent, à cet effet, une importante opération d'afféagement ${ }^{5}$ des terres, spéculant sur la propriété foncière pour ranimer l'ardeur des paysans bellilois durement éprouvés par les années de guerre ${ }^{6}$. Profitant de cette grande redistribution des sols et du bouleversement d'un paysage agraire séculaire, les États de Bretagne envisagent alors d'installer une communauté acadienne à Belle-lle. Mais si cette opération soutenue par une ferme volonté politique et d'importants moyens financiers apparaît d'emblée séduisante, elle n'en demeure pas moins difficile à réussir. De fait, elle conduit à rassembler deux groupes sociaux à la sensibilité exacerbée par les conflits et aux identités différentes, sur des terres à la propriété desquelles la population insulaire aspire légitimement. Nous souhaitons présenter ici les modalités humaines et matérielles de cette tentative d'intégration, originale et atypique, dans une île bretonne, à la fin du règne de Louis XV.

\section{Les conditions matérielles de l'installation}

\section{L'étape préparatoire}

Le projet d'établir des familles acadiennes à Belle-Île prend forme au début de l'été 1763, alors que viennent à peine d'arriver à Morlaix et à SaintMalo plusieurs navires d'exilés ${ }^{7}$. La Province, qui se lance au même moment dans la reconstruction totale de l'île, veut profiter de cette occasion pour insérer plus aisément une partie de cette communauté du "Nouveau Monde ». L'idée semble d'autant plus judicieuse que l'afféagement doit conduire à une redistribution générale des terres et à de lourds investissements structurels dans l'ensemble des campagnes. Par ailleurs, les instances provinciales estiment que l'intégration d'une population extrainsulaire à Belle-l̂le est propre à pallier l'effondrement démographique issu de la crise, et plus encore à contribuer à une émulation rurale. En juillet, une lettre adressée par la Commission des domaines au duc d'Aiguillon confirme précisément cette analyse ${ }^{8}$ :

" [...] Nous désirons, Monsieur, que l'établissement des Acadiens à BelleIsle procure des avantages considérables tant pour la population que pour la

4. Par un contrat d'échange signé le 18 février 1759, le roi a concédé l'usage de cette partie de son domaine en "l'engageant aux États de Bretagne ". Le substantif " engagiste " est utilisé alors pour désigner le contractant.

5. Le terme d'afféagement désigne, en Bretagne, la concession seigneuriale de terres généralement en friche; l'opération est en fait un accensement.

6. CERINO, Christophe, "Les paysans de la mer et l'événement. Destructions, misères et reconstruction des campagnes à Belle-Île-en-Mer au lendemain de la guerre de Sept Ans ", Enquêtes Rurales, n ${ }^{\circ}$ 7, 2000, p. 97-114.

7. Arch. dép. d'Ille-et-Vilaine, C 5156.

8. Arch. dép. d'Ille-et-Vilaine, C 5127. 
culture des terres : leur industrie, leur amour pour le travail, pourraient inspirer de l'émulation aux anciens colons de Belle-Isle qui sont naturellement lents et paresseux, et qui négligent la culture de la plus grande partie de leurs terres pour y faire la pêche de la sardine..."

Ce projet reçoit le plein accord des Acadiens, dont certains représentants sont venus à Belle-Île en prendre la mesure, et aboutit au cours du mois de décembre, encouragé par Choiseul qui s'engage à financer leur installation. À cet effet, l'abbé Le Loutre, ancien vicaire général du diocèse de Québec, reçoit de la part du Ministre la mission de veiller au bon déroulement de l'opération.

Deux années, occupées en tergiversations diverses pour obtenir des crédits et aplanir les difficultés de mise en œuvre, séparent la décision administrative de l'arrivée des premières familles. Un des problèmes majeurs, parmi ceux qui surgissent alors, est de savoir comment et où implanter les Acadiens, sachant qu'ils doivent s'intégrer et être acceptés par la communauté insulaire. Cette question fondamentale apparaît d'autant plus complexe qu'elle est posée pendant la phase initiale de remise en culture des terres lancée par la Province dès l'été 1763. En effet, comment demander à des paysans bellilois, durement éprouvés par la crise agraire et l'occupation anglaise, de remettre en culture des parcelles que l'on concédera probablement ensuite à des Acadiens ${ }^{9}$ ?

En concertation avec l'abbé Le Loutre et le baron de Warren ${ }^{10}$, l'inspecteur des Domaines Isambert, chargé de coordonner la reconstruction à Belle-Île, joue alors un rôle crucial, émettant diverses propositions sous le regard attentif de la Province. Prônant une dissémination des familles dans l'île afin de provoquer les contacts et l'intégration, il récuse toute logique de regroupement paroissial ou villageois. Pourtant, après lecture de diverses suggestions, les États de Bretagne préconisent dans leur délibération du 5 mars 1765 de ne pas placer d'Acadiens à Sauzon, préférant...

«[...] L'extrémité des trois paroisses [de Palais, Bangor et Locmaria], à commencer à l'endroit où elles se réunissent, et de proche en proche autant que faire se pourra, sans forcer les anciens habitants à quitter leurs habitations, à moins qu'ils n'y consentissent de gré à gré ${ }^{11} \ldots$ "..

À la fin du mois, dans l'impossibilité d'aboutir à un consensus local sur cette disposition, Isambert élabore une nouvelle solution, qui utiliserait au maximum les villages détruits pendant la guerre. Tout en maintenant le principe de dissémination, ce projet a également le mérite de limiter les déplacements de paysans bellilois. Suivant ses prescriptions, la Commission des Domaines refuse alors même de concéder intégralement aux Acadiens les hameaux de Kerguénolé et de Parlavan, comme le réclamait l'abbé Le Loutre ${ }^{12}$. Finalement, une quarantaine de villages choisis au

9. Arch. dép. d'Ille-et-Vilaine, C 5143.

10. Commandant militaire de Belle-Île de 1763 à 1775.

11. Arch. dép. du Morbihan, 4-A. 3.

12. Arch. dép. d'Ille-et-Vilaine, C. 5128. 
sein des quatre paroisses sont retenus pour établir les familles, selon l'implantation suivante ${ }^{13}$.

\section{L'installation acadienne à Belle-Île-en-Mer (1766)}

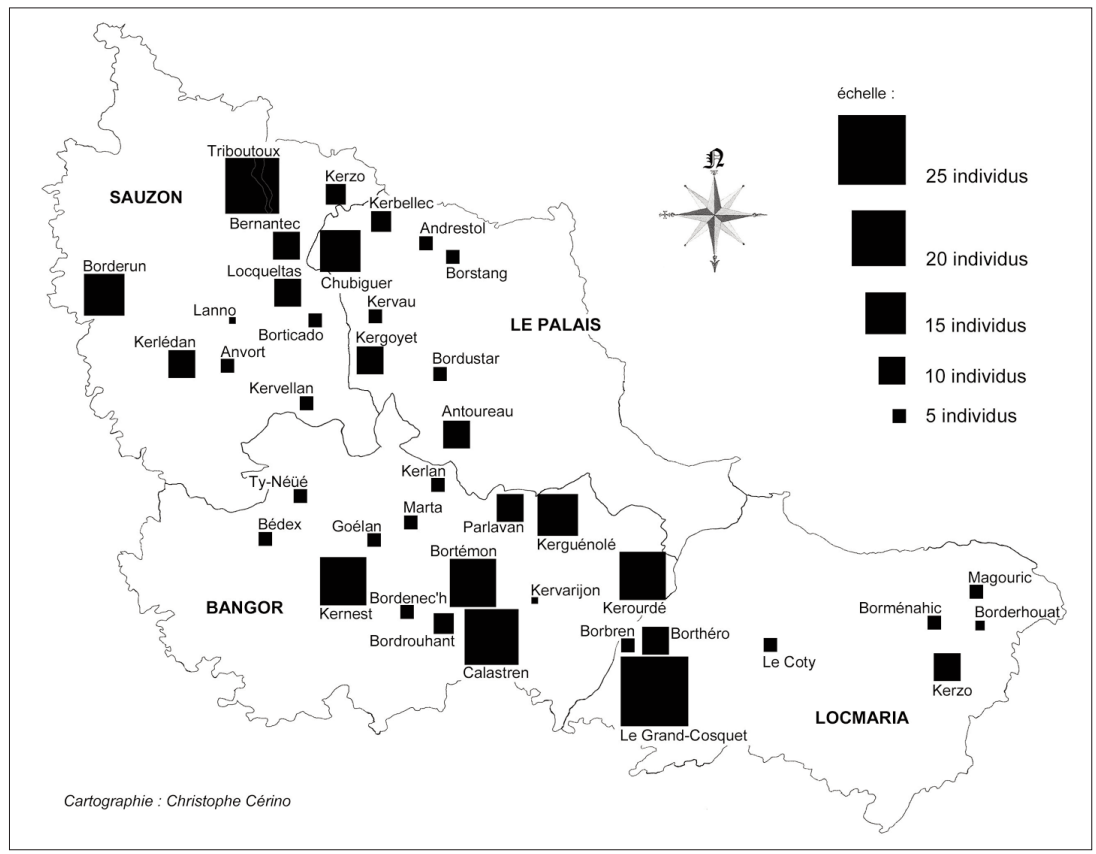

À la fin de 1765, l'arrêt des dispositions préliminaires à l'afféagement confirme donc la volonté d'éparpiller systématiquement la communauté acadienne, dans le but de favoriser son intégration à la population belliloise.

\section{Les données matérielles de l'établissement}

Au début du mois de novembre 1765, 354 exilés débarquent au Palais, conduits par l'abbé Le Loutre. Ils sont bientôt rejoints par six autres personnes tandis qu'on lieu les trois premières naissance à Belle-Île. L'effectif du groupe atteint au total 363 individus répartis en 78 familles ${ }^{14}$. Mais le retard pris par les opérations d'afféagements pose alors des problèmes d'installation dans les villages. Ainsi, après une déportation et une mise en résidence surveillée particulièrement pénible en Angleterre, ces malheureux doivent encore attendre près d'une année avant de retrouver l'inti-

13. Arch. dép. d'Ille-et-Vilaine, C. 5158 (Carte de synthèse effectuée à partir d'un traitement sur système de gestion de base de données).

14. Arch. dép. du Morbihan, 4-A. 1. 
mité d'un foyer et plus encore d'envisager leurs premières récoltes. Dans l'attente de la distribution des terres, la plupart des Acadiens restent donc cantonnés dans la ville du Palais où ils sont logés notamment dans la précarité des magasins de la seigneurie...

De fait, compliquée par des problèmes techniques (relance de l'activité agricole, mise en place du cadre juridique de la réforme foncière, réalisation complexe des arpentages) et humains (inquiétudes et mécontentements de la population belliloise, mise en cause de l'inspecteur Isambert) ${ }^{15}$, l'attribution des concessions n'intervient pas avant l'automne 1766. En respectant scrupuleusement les dispo-

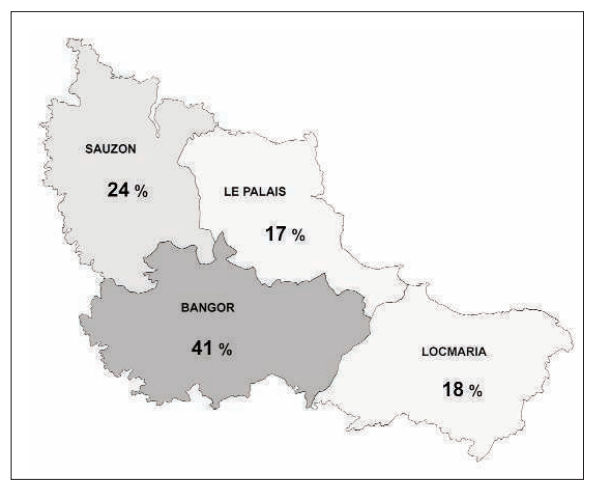
sitions préliminaires, les commissaires provinciaux veillent alors à installer dans chaque paroisse des familles acadiennes, qui comptent à présent 383 individus ${ }^{16}$, soit $8 \%$ de la population insulaire, répartis de la sorte ${ }^{17}$ : Bangor : 41 \%; Sauzon : 24 \% ; Locmaria : 18 \% ; Le Palais : $17 \%$.

Les conditions d'établissement de ces familles sont particulièrement éprouvantes, même si elles peuvent compter sur un soutien financier non négligeable de l'État, qui verse à chaque rapatrié une indemnité quotidienne. Ainsi, l'installation tardive dans les villages contraint d'emblée les Acadiens à mener d'épuisants labeurs pour construire à la hâte leurs maisons avant l'arrivée de l'hiver. Ils les poursuivent pendant toute l'année 1767 pour continuer la réalisation des fermes et pour commencer à labourer des terres, dont la plupart sont en friche depuis l'occupation anglaise! En effet, pour limiter les sources de conflit, les commissaires provinciaux ont évité le plus possible d'octroyer aux Acadiens des parcelles qui avaient été rendues à la culture par les paysans bellilois. En tout état de cause, les premières récoltes ne peuvent donc pas être sérieusement envisagées avant 1768, soit presque trois ans après l'arrivée à Belle-llle des rapatriés ${ }^{18}$ !

En revanche, les moyens financiers consacrés à l'installation des familles acadiennes sont à la hauteur des besoins. Au total, 56000 livres sont octroyées par Louis XV, représentant près de 30 \% du budget de la reconstruction de toute l'île ${ }^{19}$ ! Outre les matières premières nécessaires à

15. CERINO, Christophe, Sociétés insulaires, guerres maritimes et garnisons. Belle-Île-enMer au siècle de Louis XV, op. cit., p. 199-225.

16. Nous recensons 20 naissances supplémentaires à compter du $1^{\mathrm{er}}$ janvier 1766 (d'après une étude effectuée par Marie-Antoinette et Daniel Rohan, généalogistes professionnels, de Bretagne-Généalogie).

17. Arch. dép. d'Ille-et-Vilaine, C 5158.

18. Arch. dép. d'Ille-et-Vilaine, C 5128.

19. Arch. dép. d'Ille-et-Vilaine, C 5169. 
l'édification des fermes, les nouveaux arrivants reçoivent ainsi cheptel, chevaux, charrettes et autres outils nécessaires à leurs exploitations et se voient gratifier d'une somme de 34 livres par foyer.

À la fin de 1767, nous pouvons considérer que les 78 familles sont enfin installées dans leurs chaumières, après une décennie de douloureuses épreuves. Sans négliger les difficultés qu'impose le redémarrage complet de leurs activités agricoles, leur sort semble alors bien plus enviable que celui de la plupart des Acadiens errant dans les ports du royaume...

\section{Une intégration réussie ${ }^{20}$ ?}

\section{Un contexte initial difficile}

En 1766, l'établissement des familles de rapatriés dans les campagnes insulaires se présente d'emblée comme une opération délicate à mener. Il s'agit pour la Province de réussir à faire se rencontrer deux populations singulières, tout en accomplissant en parallèle une grande réforme foncière.

La communauté acadienne arrive à Belle-lle avec un lourd passif de souffrances. La plupart de ses membres sont originaires de la région des Mines, constituée par les paroisses de La Rivière-aux-Canards, de Grand-Pré, et de Piziquid, qui ont particulièrement résisté aux troupes britanniques. Ils constituent ensuite une part des 1500 déportés dont la Virginie a refusé l'immigration proposée par le Gouvernement anglais. Ces Acadiens ont par conséquent été exilés en Angleterre et placés en résidence surveillée pendant toute la guerre de Sept Ans, principalement dans les villes de Falmouth, Liverpool, et Southampton ${ }^{21}$. Ces événements douloureux, conduisant à de fortes solidarités de groupe, contribuent assurément à renforcer l'identité de cette population du " Nouveau Monde ». Bien entendu, les contextes géographique, politique et économique dans lesquels ont vécu les colons d'Amérique participent aussi de ces critères identitaires.

La société belliloise, pour sa part, se décompose schématiquement entre une population rurale vivant repliée dans ses exploitations agricoles et une communauté citadine ouverte aux contacts grâce à ses activités maritimes (pêche côtière et négoce sardinier), commerciales, et plus encore par suite de la présence permanente d'une garnison depuis la seconde moitié du XVII ${ }^{\mathrm{e}}$ siècle. Cette confrontation avec des troupes royales venues d'un large horizon géographique et les échanges économiques bénéfiques qui en découlent, donnent aux Palatins une propension à l'ouverture plus importante que pour les autres paroisses de l'île, où la présence de soldats n'est effective qu'en temps de guerre, suivant des logiques

20. Cette étude ne tend qu'à évoquer succinctement la situation des Acadiens installés à Belle-Île, une décennie après l'afféagement. Une recherche sur l'intégration économique et sociale de cette communauté reste à effectuer sur la longue durée.

21. Daligaut, Marguerite, "Les Acadiens en Angleterre (1755-1763) ", Bulletin de l'Association pour l'Histoire de Belle-Île, $\mathrm{n}^{\circ}$ 3, 1964, p. 5-6. 
de soumission aux corvées militaires ${ }^{22}$. Or, c'est précisément le monde rural, a priori plus méfiant à l'égard des étrangers, qui est concerné par l'implantation de 78 familles de rapatriés.

Les paysans qui vont être en contact direct avec les Acadiens sont susceptibles de partager avec eux les tâches professionnelles agricoles, les souffrances émanant de la guerre et de l'occupation britannique, voire les sentiments anglophobes qui pourraient en découler. Pour le reste, l'univers d'étroit contrôle seigneurial et royal dans lequel ils ont toujours vécu, l'ancrage des familles dans les paroisses insulaires, la culture et la langue bretonnes constituent autant d'éléments de divergences identitaires à prendre en considération. Ainsi, la misère profonde de ces deux communautés n'apparaît pas d'emblée comme un vecteur de convergence, propre à créer une solidarité commune, mais plutôt comme un catalyseur d'inquiétudes. Dans ce contexte délicat, la population rurale belliloise ne peut être que méfiante, voire totalement hostile, à l'idée de devoir partager une terre dont elle attend la propriété depuis toujours.

Au printemps 1766, dans une action qui se situe à l'opposé de sa mission de concorde sociale, le recteur de Bangor s'emploie à rassembler les mécontentements contre les Acadiens et à s'en faire l'émissaire retors auprès des instances provinciales. Vexé de ne pas jouer un rôle de premier plan dans le processus d'afféagement et particulièrement pugnace envers les agents commis pour cette fonction, il accuse alors Isambert et l'abbé Le Loutre d'agir de concert contre les intérêts bellilois ${ }^{23}$. En mai 1766, tandis que se pose l'embarrassante question du déplacement de certains ruraux, Le Sergent lance une offensive sournoise contre les familles de rapatriés, écrivant à la Commission des Domaines ${ }^{24}$ :

"[...] Il ne paraît pas que ces nouveaux colons fassent jamais des bons laboureurs pour le païs-cy. C'est le sentiment de tous les recteurs et prêtres et de tous les habitans de l'Isle qui les voyent travailler quelques petits jardinets..."

Plus grave encore, il prétend rapporter de ses paroissiens les propos suivants :

"[...] Les Acadiens sont-ils donc venus à Belle-Isle pour nous précipiter dans tous les malheurs et pour faire exercer contre nous des cruautés et des hostilités telles que nous n'en avons pas souffert de la part des Anglais [...]. C'est nous forcer de rester exposés aux injures du temps ou de sortir d'une isle que nous avons défendue au péril de nos vies et qui est notre patrie, pour y loger des étrangers..."

Et il conclut une de ses correspondances par :

22. CERINO, Christophe, "Sociétés insulaires et garnisons : Relations et regards de deux communautés à Belle-Île-en-Mer au XVIII ${ }^{\mathrm{e}}$ siècle ", Représentations et images du littoral, Rennes, PUR, 1998, p. 47-55.

23. Arch. dép. d'Ille-et-Vilaine, C 5143.

24. Arch. dép. d'Ille-et-Vilaine, C 5145. 
"[...] La balance doit pencher d'un côté ou de l'autre... "

Entre l'inquiétude profonde des îliens, les intrigues habituelles du recteur, et la confusion générale issue de la première phase de reconstruction des campagnes, il convient bien entendu de faire la part des choses. L'acceptation des familles acadiennes n'est certainement pas facile pour les Bellilois, installés dans leurs exploitations depuis plusieurs générations, et dont la tendance à la xénophobie est plus que probable. Par ailleurs, même s'il est prévu de limiter au maximum le déplacement des autochtones, la grande redistribution des terres implique forcément d'importantes perturbations locales, sources de tensions plus ou moins contenues. Dans ce contexte défavorable, nous imaginons donc les difficultés de communication entre les deux communautés, renforcées par la barrière linguistique pour les paysans qui ne parlent que le Breton!

Au-delà d'inimitiés plus ou moins affichées en fonction des individus, les conflits se traduisent par des refus d'évacuer les parcelles comme à Antoureau, Chubiguer et Kerzo " où les colons s'obstinèrent à faire leur paillé sur un terrain qui avait été désigné aux Acadiens ", contraignant les commissaires provinciaux à faire procéder à quelques expulsions forcées de Bellilois de mauvaise composition ${ }^{25}$. Isambert reconnaît lui-même que " la division des terrains entre les anciens colons et les Acadiens a fait nâ̂tre des difficultés multipliées de la part des premiers qui ont toujours vu d'un vieil oeil de jalousie l'établissement de ces nouveaux colons dans l'Isle..."

Ces difficultés se poursuivent jusqu'au début de l'année 1768, à propos des contestations relatives à des chemins qui traversaient leurs exploitations et dont, en toute méconnaissance des habitudes locales, certains Acadiens ont barré les accès.

Ainsi, l'installation des 78 familles acadiennes se déroule dans un contexte difficile, tant en raison des profonds bouleversements du monde rural que de l'ouverture qui est imposée à ce dernier. Pour autant, quels que soient les problèmes rencontrés par les rapatriés d'Acadie, ils peuvent compter pendant toute cette période sur le soutien de l'État, de la Province, de personnalités locales influentes comme Isambert ou le baron de Warren, et bien entendu sur l'abbé Le Loutre. Par ailleurs, les grands travaux effectués dans les campagnes, jusqu'à la fin des années 1760, créent d'importants besoins en main-d'œuvre, propres à susciter des rapprochements d'entraide. Sans trop spéculer sur cette hypothèse, qui se fonde sur l'aspect pratique des tâches rurales, elles apparaissent néanmoins comme les meilleures occasions de rencontre des communautés.

25. Arch. dép. du Morbihan, 4-A. 3. 


\section{Bilan sommaire vers 1776}

Nous souhaitons clore cette étude par un premier diagnostic de l'intégration acadienne vers 1776. Cette limite décennale paraît d'autan plus pertinente qu'elle correspond aux termes mêmes des contrats d'afféagement qui interdisaient toutes ventes des concessions avant cette période probatoire. Elle constitue, de fait, un critère d'évaluation pertinent pour dénombrer les familles qui préfèrent abandonner Belle-Île au début du règne de Louis XVI.

Au total, seulement quatorze d'entre elles se débarrassent de leurs exploitations entre 1776 et 1778, soit $18 \%$ des rapatriés installés dix ans plus tôt. La plupart de ces personnes retournent en Amérique, soit directement, soit quelques années plus tard, à l'instar d'Honoré Duon qui, après un séjour à Nantes, s'embarque pour la Louisiane en $1785^{26}$. Il paraît difficile d'apprécier de façon tangible les motivations de ces départs. Nous pouvons tout au plus supposer que la nostalgie du territoire natal, la mauvaise qualité de certaines concessions, l'incapacité à résister aux difficultés économiques du début des années 1770 et quelques problèmes locaux d'assimilation se sont conjugués pour motiver ces nouvelles expatriations.

En revanche, il semble que l'intégration matérielle et économique de la plupart des familles restantes soit déjà bien avancée. À titre d'exemple, d'après son inventaire, Charles Le Blanc laisse 650 livres de biens divers en 1772. Si cette somme ne le place pas au rang des meilleurs afféagistes, il n'en atteste pas moins d'une situation supérieure à celle de bien des Bellilois de souche ${ }^{27}$. De même, quelques années plus tard, la succession de Jean Granger indique que son foyer a déjà atteint le stade d'un " confort " élémentaire avec 1288 livres de biens déclarés ${ }^{28}$. Sur le plan social, quelques indicateurs permettent de pressentir l'amorce d'une intégration. C'est notamment en ces termes que nous interprétons les $15 \%$ de mariages acadiens qui ont été contractés avec des Bellilois(es) entre 1769 et $1789^{29}$. Même si la majorité des exilés continuent à s'unir entre eux, ces quelques alliances constituent un signe d'ouverture non négligeable.

Par ailleurs, l'examen des sources judiciaires ne révèle pas de conflits majeurs ni de violences rurales, une fois réglées les difficultés propres à la mise en œuvre de l'afféagement. Il nous semble là encore possible d'interpréter positivement le silence des archives. En effet, en cas de problèmes, le seul recours pour ces familles de rapatriés isolées dans les villages, c'est précisément la saisine de l'arbitrage judiciaire. Or, nous ne relevons rien de tel dans les documents de la sénéchaussée, hormis deux affaires pour injures émises par Pierre Deline à l'encontre de Jean Tirnay et Pélagie Hébert,

26. Daligaut, Marguerite, "Les départ d'Acadiens ", Bulletin de l'Association Historique de Belle-Ile, ${ }^{\circ}$ 8, 1965, p. 5 .

27. Arch. dép. du Morbihan, B. 2167. De Kerourdé en Bangor.

28. Arch. dép. du Morbihan, B 2155. D’Andrestol en Le Palais.

29. D'après une enquête effectuée dans les registres de catholicité sur l'ensemble des mariages de la communauté acadienne. 
ses voisins de Kervarijon (Bangor), qui tout comme lui sont d'origine... acadienne $^{30}$. Enfin, au plan politique, signalons que Jo-Simon Granger entre au " général de paroisse " en 1782 et y devient même " égailleur " de l'impôt.

En conclusion, les efforts consentis par l'État et la Province pour l'installation des Acadiens à Belle-lle-en-Mer ont abouti de façon positive, en dépit d'un contexte initial difficile. Sans sous-estimer les nombreux problèmes posés par la confrontation avec la population rurale insulaire, il ya bel et bien eu une lente mais effective intégration, comme le démontrent certains indicateurs. D'une part, l'installation de 1766 a forcé la population belliloise à une acceptation " de l'autre ", favorisant pour le monde paysan une certaine ouverture, tout à fait atypique dans une île bretonne de l'Atlantique. D'autre part, à la veille de la Révolution, les deux tiers des familles acadiennes installées en 1766 travaillent encore dans leurs exploitations, et ceci malgré les difficultés économiques du début des années 1770. Notons, par ailleurs, que leur lutte pour survivre et leurs bonnes connaissances agronomiques ont incontestablement contribué au succès global de l'afféagement. Par comparaison, tout en relativisant les échelles démographiques (1472 Acadiens contre 363), l'autre implantation de rapatriés qui fut tentée dans le Poitou, entre 1773 et 1776, s'est soldée par un réel échec. De fait, à la fin du XVIII ${ }^{\mathrm{e}}$ siècle, la plupart des Acadiens ont fini par retourner en Amérique ${ }^{31} \ldots$

\section{RESUME}

Aux lendemains de la guerre de Sept Ans, le projet d'installation à BelleÎle-en-Mer de 78 familles acadiennes, dans le cadre de la reconstruction des campagnes dévastées par le conflit, s'impose d'emblée aux instances provinciales comme une opération délicate à réaliser. Au-delà des problèmes matériels qu'elle présente, cette expérience originale conduit à l'intégration, atypique dans une île bretonne, d'une communauté rapatriée des confins du royaume.

\section{ABSTRACT}

In the wake of the Seven Year War, the project of making 78 Acadian families settle in the southern Brittany island of Belle-Ille-en-Mer, with a view to rebuilding the countryside which had been ravaged by the war, turns out to be a rather touchy operation for the state of Britanny to carry out. Beyond the material problems that it introduces, this unprecedented experiment leads to atypical integration of a community gathered up from the remotest comers of the kingdom.

30. Arch. dép. du Morbihan, B. 2153-2154.

31. Theriault, Léon, "L'Acadie de 1763 à 1978 ", Les Acadiens des Maritimes, Université de Moncton, Centre d'études acadiennes, 1980, p. 49 à 93. 